\title{
THE CUBIC SPHERICAL OPTIMIZATION PROBLEMS
}

\author{
XINZHEN ZHANG, LIQUN QI, AND YINYU YE
}

\begin{abstract}
In this paper, the cubic spherical optimization problems, including the cubic one-spherical/two-spherical/three-spherical optimization problems, are discussed. We first show that the two-spherical optimization problem is a special case of the three-spherical optimization problem. Then we show that the one-spherical optimization problem and the two-spherical optimization problem have the same optimal value when the tensor is symmetric. In addition, NP-hardness of them are established. For the cubic three-spherical optimization problem, we discuss the conditions under which the problem is polynomial time solvable and if the polynomial time approximation scheme (PTAS) exists. Then we present a relative quality bound by finding the largest singular values of matrices. Finally, a practical method for solving the cubic three-spherical optimization problem is proposed and preliminary numerical results are reported.
\end{abstract}

\section{INTRODUCTION}

The cubic one-spherical optimization problem has the following form:

$$
\begin{array}{ll}
\min _{x \in \Re^{n}} & f(x):=\mathcal{A} x^{3}=\sum_{i, j, k=1}^{n} a_{i j k} x_{i} x_{j} x_{k} \\
\text { s.t. } & \|x\|=1,
\end{array}
$$

where $n \geq 2$ and $\mathcal{A}$ is a third order $(n \times n \times n)$-dimensional real symmetric tensor. Here, tensor $\mathcal{A}$ is symmetric in the sense that its element $a_{i j k}$ is invariant under any permutation of their indices $(i, j, k)$. In some earlier literature [5, 7, 12, symmetric tensor is also called being supersymmetric.

The cubic two-spherical optimization problem has the following form:

$$
\begin{array}{cl}
\min _{x \in \Re^{n}, z \in \Re^{q}} & g(x):=\mathcal{B} x^{2} z=\sum_{i, j=1}^{n} \sum_{k=1}^{q} b_{i j k} x_{i} x_{j} z_{k} \\
\text { s.t. } & \|x\|=1,\|z\|=1,
\end{array}
$$

where $n, q \geq 2$ and $\mathcal{B}$ is a third order $(n \times n \times q)$-dimensional real partially symmetric tensor. Tensor $\mathcal{B}$ is said to be partially symmetric in the sense that $b_{i j k}=b_{j i k}$, for all $i, j=1,2, \cdots, n$, and $k=1,2, \cdots, q$.

Received by the editor June 4, 2009 and, in revised form June 2, 2011.

2010 Mathematics Subject Classification. Primary 15A18, 15A69, 90C60.

Key words and phrases. Cubic spherical optimization, approximation solution, polynomial time approximation scheme (PTAS).

The first author is supported by the National Natural Science Foundation of China (Grant Nos. 11101303 and 11171180), and Independent Innovation Fund of Tianjin University.

The second author is supported by the Hong Kong Research Grant Council. 
The cubic three-spherical optimization problem has the following form:

$$
\begin{array}{cl}
\min _{x \in \Re^{n}, y \in \Re^{p}, z \in \Re^{q}} & h(x):=\mathcal{C} x y z=\sum_{i=1}^{n} \sum_{j=1}^{p} \sum_{k=1}^{q} c_{i j k} x_{i} y_{j} z_{k} \\
\text { s.t. } & \|x\|=1,\|y\|=1,\|z\|=1,
\end{array}
$$

where $n, p, q \geq 2$ and $\mathcal{C}$ is a third order $(n \times p \times q)$-dimensional real tensor.

These three problems arise from the best rank-one approximation to $\mathcal{A}, \mathcal{B}$ and $\mathcal{C}$, respectively. In signal processing, a discrete multidimensional signal is treated as an $m$ th order tensor with $m \geq 3$ and the optimal low-rank approximation of tensor is used to approximate of multidimensional signal. When $m=3,(1.3)$ is used as a suboptimal solution of low-rank approximation of tensor in [9]. In [15], the optimal value of (1.1) with $n=3$ is used to characterize the phase of the magnetic resonance signal in biological tissues. The best rank-1 approximation of higher order tensor has some applications in image processing and wireless communication systems, etc., 3 , 2, 5, 6, 7, 14. Some other applications are about the eigenvalues of tensors; see [12, 13].

Furthermore, these three problems are homogeneous polynomial optimization problems, which have been considered by many scholars. In [14], some computational methods for solving (1.3) were proposed. In [11, minimizing the homogeneous polynomial over multi-spheres and unit-spheres was discussed there and some bounds were presented via sum of squares (SOS) relaxation. In [4, the authors proved the NP-hardness of the multi-variate homogeneous polynomial function and proposed approximation algorithms for considered problems.

Contributions. First, we show that the cubic one-spherical/two-spherical optimization problems are special cases of the cubic three-spherical optimization problem, which can be used to improve the bound presented by Theorem 4.4 in [1] when $d=2$. Then we reformulate the cubic two-spherical optimization problem as an NP-hard quartic optimization problem over a unit sphere. Based on this reformulation, the NP-hardness of the cubic two-spherical/three-spherical optimization problems are established. These will be presented in Section 2 .

In the subsequent discussion, we only focus on the cubic three-spherical optimization problem. We show that when some matrices are simultaneously diagonalized and $\min \{n, p, q\}=2$, the problem is polynomial time solvable. Then some PTAS cases are discussed by grid sampling and SOS. These will be discussed in Section 3 and Section 4.

Last, we present a quality bound for (1.3) by finding the largest singular values of some matrices in Section 5. Based on the quality bound, we propose a practical method for solving (1.3) and report some preliminary results in the last section.

\section{RELATIONShips of THESE PROBLEMS AND THEIR NP-HARDNESS}

When the tensor involved in (1.3) is symmetric, is the optimal value of (1.3) equal to the optimal value of (1.1)? In other words, what are the relationships of the considered problems? In this section, we answer the question clearly.

To answer this question, we first show that (1.3) can be equivalently reformulated as (1.2) in polynomial time when the tensor is partially symmetric.

Theorem 2.1. Suppose that tensor $\mathcal{C}$ is a third order $(n \times n \times q)$-dimensional partially symmetric tensor with respect to the first two indices. Then there exist $x^{*} \in \Re^{n}$ and $z^{*} \in \Re^{q}$ such that $\left(x^{*}, x^{*}, z^{*}\right)$ is a global minimizer of (1.3). 
Proof. Let $(\bar{x}, \bar{y}, \bar{z})$ be an optimal solution of (1.3) with optimal value $\lambda \leq 0$. It is easy to see that $(\bar{x}, \bar{y}, \bar{z})$ is a KKT point of (1.3), which indicates that there exist $\alpha, \beta$ and $\gamma$ such that

$$
\left\{\begin{array}{l}
\sum_{j=1}^{n} \sum_{k=1}^{q} c_{i j k} \bar{y}_{j} \bar{z}_{k}=\alpha \bar{x}_{i}, \quad i=1, \cdots, n, \\
\sum_{i=1}^{n} \sum_{k=1}^{q} c_{i j k} \bar{x}_{i} \bar{z}_{k}=\beta \bar{y}_{j}, \quad j=1, \cdots, n, \\
\sum_{i=1}^{n} \sum_{j=1}^{n} c_{i j k} \bar{x}_{i} \bar{y}_{j}=\gamma \bar{z}_{k}, \quad k=1, \cdots, q, \\
\|\bar{x}\|=1, \\
\|\bar{y}\|=1, \\
\|\bar{z}\|=1 .
\end{array}\right.
$$

Then,

$$
\alpha=\alpha \sum_{i=1}^{n} \bar{x}_{i}^{2}=\sum_{i=1}^{n} \sum_{j=1}^{n} \sum_{k=1}^{q} c_{i j k} \bar{x}_{i} \bar{y}_{j} \bar{z}_{k}=\lambda .
$$

Similarly, $\beta=\lambda$ and $\gamma=\lambda$. Thus, there holds

$$
\left\{\begin{array}{l}
\sum_{j=1}^{n} \sum_{k=1}^{q} c_{i j k} \bar{y}_{j} \bar{z}_{k}=\lambda \bar{x}_{i}, \quad i=1, \cdots, n, \\
\sum_{i=1}^{n} \sum_{k=1}^{q} c_{i j k} \bar{x}_{i} \bar{z}_{k}=\lambda \bar{y}_{j}, \quad j=1, \cdots, n, \\
\sum_{i=1}^{n} \sum_{j=1}^{n} c_{i j k} \bar{x}_{i} \bar{y}_{j}=\lambda \bar{z}_{k}, \quad k=1, \cdots, q, \\
\|\bar{x}\|=1 \\
\|\bar{y}\|=1 \\
\|\bar{z}\|=1 .
\end{array}\right.
$$

We denote $M=\mathcal{C} \bar{z}$ as a real $n \times n$ symmetric matrix with its $(i, j)$ th entry being $M_{i j}=\sum_{k=1}^{q} c_{i j k} \bar{z}_{k}$. Then, (2.1) leads to

$$
\left\{\begin{aligned}
M(-\bar{y}) & =-\lambda \bar{x}, \\
M \bar{x} & =-\lambda(-\bar{y}), \\
\|\bar{x}\|=1, & \|-\bar{y}\|=1,
\end{aligned}\right.
$$

which indicates that $\bar{x},-\bar{y}$ are left-singular and right-singular vectors for $-\lambda$ of $M$, respectively. From the symmetric property of matrix, we know that either $\lambda$ or $-\lambda$ is the eigenvalue of $M$. Now we consider two cases.

Case 1. If $\lambda$ is an eigenvalue of $M$ associated with an eigenvector $x^{*}$, we denote $z^{*}=\bar{z}$. Then, $\mathcal{C} x^{*} x^{*} z^{*}=\lambda$.

Case 2. If $-\lambda$ is an eigenvalue of $M$ associated with an eigenvector $x^{*}$, we denote $z^{*}=-\bar{z}$. Then, $\mathcal{C} x^{*} x^{*} z^{*}=\lambda$.

Therefore, $\left(x^{*}, x^{*}, z^{*}\right)$ is a global minimizer of optimization problem (1.3) and the proof is completed.

Now we are ready to show that (1.1) and (1.2) have the equal optimal values when $\mathcal{B}$ is symmetric. To show this result, we need the following discussion. 
Algorithm 2.1.

- Initial Step: Input symmetric tensor $\mathcal{B}$ and an optimal solution $\left(x^{(0)}, z^{(0)}\right)$ satisfying

$$
\mathcal{B}\left(x^{(0)}\right)^{2} z^{(0)}=\lambda \leq 0,\left(x^{(0)}\right)^{\top} z^{(0)} \geq 0
$$

Let $t=0$.

- Iterative Step: If $x^{(t)}=z^{(t)}$, Stop; Otherwise, let matrix $M^{(t)}=\mathcal{B} x^{(t)}$ with its entries $\left(M^{(t)}\right)_{j k}=\sum_{i=1}^{n} b_{i j k} x_{i}^{(t)}$. Define

$$
x^{(t+1)}=\frac{x^{(t)}+z^{(t)}}{\left\|x^{(t)}+z^{(t)}\right\|}, z^{(t+1)}=x^{(t)} .
$$

Let $t:=t+1$.

Lemma 2.1. The Algorithm 2.1 is well defined.

Proof. If $\left(x^{(0)}, z^{(0)}\right)$ is an optimal solution of (1.2), then $\left(-x^{(0)}, z^{(0)}\right)$ is also an optimal solution of (1.2). So the assumption that $\left(x^{(0)}\right)^{\top} z^{(0)} \geq 0$ is reasonable.

Lemma 2.2. Suppose that Algorithm (2.1) terminates in a finite number of steps, then there exists $x^{*}$ such that $\left(x^{*}, x^{*}\right)$ is an optimal solution of (1.2).

Proof. At first, we show that for all $t,\left(x^{(t+1)}, z^{(t+1)}\right)$ is an optimal solution of (1.2).

Let $\left(x^{(t)}, z^{(t)}\right)$ be an optimal solution of (1.2) and $x^{(t)}-z^{(t)} \neq 0$. From the definition of $M^{(t)}$, we have

$$
\begin{cases}M^{(t)} x^{(t)} & =\lambda z^{(t)} \\ M^{(t)} z^{(t)} & =\lambda x^{(t)}\end{cases}
$$

This indicates that $M^{(t)}\left(x^{(t)}+z^{(t)}\right)=\lambda\left(x^{(t)}+z^{(t)}\right)$, which implies that $x^{(t)}+z^{(t)}$ is an eigenvector of $M^{(t)}$ with eigenvalue $\lambda$. So

$$
\left(\frac{x^{(t)}+z^{(t)}}{\left\|x^{(t)}+z^{(t)}\right\|}\right)^{\top} M^{(t)}\left(\frac{x^{(t)}+z^{(t)}}{\left\|x^{(t)}+z^{(t)}\right\|}\right)=\lambda,
$$

that is to say,

$$
\mathcal{A}\left(\frac{x^{(t)}+z^{(t)}}{\left\|x^{(t)}+z^{(t)}\right\|}\right)^{2} x^{(t)}=\lambda .
$$

Therefore, we can assert that $\left(x^{(t+1)}, z^{(t+1)}\right)$ is also an optimal solution of (1.2).

If Algorithm 2.1 terminates in a finite number of steps, there exists $t$ such that $x^{(t)}-z^{(t)}=0$. Let

$$
x^{*}=x^{(t)} .
$$

The proof is completed.

Lemma 2.3. Suppose that $\left(x^{*}, z^{*}\right)$ is an accumulation point of the infinite sequence $\left\{\left(x^{(t)}, z^{(t)}\right)\right\}$ generated by Algorithm 2.1, Then either $\left(x^{*}, x^{*}\right)$ or $\left(-x^{*},-x^{*}\right)$ is an optimal solution of (1.2). 
Proof. As shown in Lemma 2.2, we know that for all $t,\left(x^{(t)}, z^{(t)}\right)$ is an optimal solution of (1.2). Moreover,

$$
\left(x^{(t+1)}\right)^{\top} z^{(t+1)}=\left(\frac{x^{(t)}+z^{(t)}}{\left\|x^{(t)}+z^{(t)}\right\|}\right)^{\top} x^{(t)}=\frac{1+\left(x^{(t)}\right)^{\top} z^{(t)}}{\left\|x^{(t)}+z^{(t)}\right\|}=\sqrt{\frac{1+\left(x^{(t)}\right)^{\top} z^{(t)}}{2}} \geq 0
$$

for all $t$. This indicates that

$$
\begin{aligned}
& \left\{\left(x^{(t+1)}\right)^{\top} z^{(t+1)}\right\}^{2}-\left\{\left(x^{(t)}\right)^{\top} z^{(t)}\right\}^{2} \\
& \quad=\frac{1}{2}\left\{2\left(x^{(t)}\right)^{\top} z^{(t)}+1\right\}\left\{1-\left(x^{(t)}\right)^{\top} z^{(t)}\right\} \\
& \geq \frac{1-\left(x^{(t)}\right)^{\top} z^{(t)}}{2} \geq 0,
\end{aligned}
$$

where the first inequality holds since $0 \leq\left(x^{(t)}\right)^{\top} z^{(t)} \leq 1$. So, we can assert that the sequence $\left\{\left(x^{(t)}\right)^{\top} z^{(t)}\right\}$ is non-decreasing.

Together with the fact that $\left\{\left(x^{(t)}\right)^{\top} z^{(t)}\right\}^{2} \leq 1$ for all $t$, we have that

$$
\lim _{t \rightarrow \infty}\left\{1-\left(x^{(t)}\right)^{\top} z^{(t)}\right\}=0 .
$$

Since $\left\|x^{(t)}\right\|=\left\|z^{(t)}\right\|=1$, there exist convergent subsequences. Without loss of generality, we assume that

$$
\left(x^{\left(t_{u}\right)}, z^{\left(t_{u}\right)}\right) \rightarrow\left(x^{*}, z^{*}\right) \text { when } t_{u} \rightarrow \infty .
$$

It is easy to see that $\left(x^{*}, z^{*}\right)$ is also an optimal solution of (1.2) and $\left(x^{*}\right)^{\top} z^{*}=1$. This leads to $x^{*}=z^{*}$ and the proof is completed.

Combining Lemma 2.3 with Theorem 2.1, we have the following result.

Theorem 2.2. If $\mathcal{C}$ is symmetric in (1.3), then there exists $x^{*}$ such that $\left(x^{*}, x^{*}, x^{*}\right)$ is an optimal solution of (1.3).

Although (1.1) can be regarded as special cases of (1.2) and (1.3), we cannot assert the NP-hardness of (1.2) and (1.3) from the NP-hardness of (1.1). This is because we do not know the complexity of Algorithm 2.1. To establish the NPhardness of the other problems, we need another reformulation of (1.2).

Theorem 2.3. For each $k$, let $B^{(k)}$ be a symmetric $n \times n$ matrix with its $(i, j)$ th element as $b_{i j k}$. Then the cubic two-spherical optimization problem (1.2) is equivalent to the following quartic maximization problem:

$$
\begin{array}{cl}
\max _{x \in \Re^{n}} & \sum_{k=1}^{q}\left\langle B^{(k)} x, x\right\rangle^{2} \\
\text { s.t. } & \|x\|=1 .
\end{array}
$$

Proof. Denote $\alpha(x)$ as a vector in $\Re^{q}$, with its $k$ th component as

$$
\alpha(x)_{k}=\sum_{i, j=1}^{n} b_{i j k} x_{i} x_{j}=x^{\top} B^{(k)} x .
$$


Then we may rewrite (1.2) as

$$
\min _{\|x\|=1} \min _{\|z\|=1}\langle\alpha(x), z\rangle=\min _{\|x\|=1}-\|\alpha(x)\|=-\max _{\|x\|=1}\|\alpha(x)\|,
$$

where the first equality holds from the Cauchy-Schwarz inequality. Furthermore, there holds

$$
\max _{\|x\|=1}\|\alpha(x)\|^{2}=\max _{\|x\|=1} \sum_{k=1}^{q}\left\langle B^{(k)} x, x\right\rangle^{2},
$$

which is problem (2.2).

As presented in Theorem 4 by Nesterov in 10, problem (2.2) is NP-hard to solve. Together with Theorem 2.1, we establish the NP-hardness of the other two problems.

In addition, we can assert that the optimal value of (1.1) can be obtained by solving (2.2). In other words, (1.1) can be equivalently reformulated as a quartic optimization problem, which improves the bound given in Theorem 4.4 in [11] when $d=2$.

In conclusion, we focus on (1.3) in the subsequent discussions.

\section{Polynomial time solvable cases of (1.3)}

In spite of NP-hardness of (1.3), there exist some special cases which can be solved in polynomial time. We present a couple of such cases in this section.

It is easy to see that $\mathcal{C} x y z$ can be rewritten as

$$
\mathcal{C} x y z=\sum_{i=1}^{n}\left(y^{\top} A^{(i)} z\right) x_{i}=\sum_{j=1}^{p}\left(z^{\top} B^{(j)} x\right) y_{j}=\sum_{k=1}^{q}\left(x^{\top} C^{(k)} y\right) z_{k},
$$

with some suitable matrices $A^{(i)} \in \Re^{p \times q}, B^{(j)} \in \Re^{q \times n}$, and $C^{(k)} \in \Re^{n \times p}$ for $i=1,2, \cdots, n, j=1,2, \cdots, p$ and $k=1,2, \cdots, q$. By using of this notation, we have the following theorem.

Theorem 3.1. Suppose that there exist orthogonal matrices $P \in \Re^{n \times n}$ and $Q \in$ $\Re^{p \times p}$ such that for all $k=1,2, \cdots, q, P^{\top} C^{(k)} Q$ are $n \times p$ diagonal matrices. Then (1.3) is polynomial time solvable.

Proof. Without loss of generality, after some orthogonal transformations, we may assume that $n \geq p$ and $C^{(k)}(k=1,2, \cdots, q)$ are $n \times p$ diagonal matrices. Then

$$
\mathcal{C} x y z=\sum_{i, j, k=1}^{n, p, q} C_{i j}^{(k)} x_{i} y_{j} z_{k}=\sum_{j=1}^{p} x_{j} y_{j} \sum_{k=1}^{q} C_{j j}^{(k)} z_{k} .
$$

Therefore, we have that

$$
\begin{aligned}
& \max _{\|x\|=1} \max _{\|y\|=1} \max _{\|z\|=1} \mathcal{C} x y z \leq \max _{\|x\|=1} \max _{\|y\|=1} \max _{\|z\|=1} \sum_{j=1}^{p} \frac{x_{j}^{2}+y_{j}^{2}}{2}\left|\sum_{k=1}^{q} C_{j j}^{(k)} z_{k}\right| \\
& =\max _{\|x\|=\|y\|=1} \sum_{j=1}^{p} \frac{x_{j}^{2}+y_{j}^{2}}{2} \max _{\|z\|=1} \sum_{k=1}^{q} C_{j j}^{(k)} z_{k} \leq \max _{\|x\|=\|y\|=1} \sum_{j=1}^{p} \frac{x_{j}^{2}+y_{j}^{2}}{2} \sum_{k=1}^{q} C_{j^{*} j^{*}}^{(k)} z_{k}^{*} \\
& =\sum_{k=1}^{q} C_{j^{*} j^{*}}^{(k)} z_{k}^{*}
\end{aligned}
$$


where $j^{*}$ and $z^{*}$ satisfy $\sum_{k=1}^{q} C_{j^{*} j^{*}}^{(k)} z_{k}^{*}=\max _{1 \leq j \leq p} \max _{\|z\|=1} \sum_{k=1}^{q} C_{j j}^{(k)} z_{k}$. Note that $j^{*}$ and $z^{*}$ can be found in polynomial time. Let $x^{*}$ and $y^{*}$ be the unit vectors such that their $j^{*}$ th entries are 1 and the rest of entries are 0 , then we have that $\max _{\|x\|=\|y\|=\|z\|=1} \mathcal{C} x y z=\mathcal{C} x^{*} y^{*} z^{*}$.

Thus we can assert that $\left(x^{*}, y^{*},-z^{*}\right)$ is an optimal solution of (1.3), which completes the proof.

Remark. If there exist $P$ and $Q$ satisfying Theorem 3.1 , then $P$ and $Q$ can be found in polynomial time. The reason for this is that $P$ and $Q$ are formed by the common singular vectors of matrix $C^{(k)}$ for all $k=1, \cdots, q$.

From the Cauchy-Schwarz inequality, it is easy to show that the cubic threespherical optimization problem (1.3) can be reformulated as the following problem:

$$
\begin{array}{cl}
\max _{x \in \Re^{n}, y \in \Re^{p}}, & h_{1}(x, y):=\|\mathcal{C} x y\|^{2} \\
\text { s.t. } & x^{\top} x=1, y^{\top} y=1 .
\end{array}
$$

In fact,

$$
h_{1}(x, y)=\sum_{k=1}^{q}\left(\sum_{i=1}^{n} \sum_{j=1}^{p} c_{i j k} x_{i} y_{j}\right)^{2}=\sum_{i, s=1}^{n} \sum_{j, t=1}^{p}\left(\sum_{k=1}^{q} c_{i j k} c_{s t k}\right) x_{i} y_{j} x_{s} y_{t} .
$$

Furthermore, by letting tensor $\tilde{\mathcal{C}}$ have its entries

$$
\tilde{c}_{i j s t}=\frac{1}{4} \sum_{k=1}^{q}\left(c_{i j k} c_{s t k}+c_{s j k} c_{i t k}+c_{i t k} c_{s j k}+c_{s t k} c_{i j k}\right)
$$

the optimization problem (1.3) will be equivalent to the bi-quadratic optimization problem, studied in 8 , with tensor $\tilde{\mathcal{C}}$. That is to say, the cubic optimization problem may be regarded as a subclass of the bi-quadratic optimization problem. According to Corollary 3.7 and Theorem 4.1 in [8], we have the following polynomial time solvable case.

Theorem 3.2. If $\min \{n, p, q\}=2$, then the cubic three-spherical optimization problem (1.3) is polynomial time solvable.

\section{PTAS OF (1.3)}

Before going further, we quote a quality measure of approximation ratio, which appeared in [8].

Definition 4.1. Let $0 \leq \epsilon<1$ and $\mathfrak{A}$ be an approximation algorithm for the minimization problem $\mathfrak{p}$. We say $\mathfrak{A}$ is a $(1-\epsilon)$-approximation algorithm if for any instance of $\mathfrak{p}$, the algorithm $\mathfrak{A}$ returns a feasible solution with its corresponding objective value $p$ such that

$$
p-p_{\min } \leq \epsilon\left(p_{\max }-p_{\min }\right),
$$

where $p_{\max }$ (resp. $p_{\min }$ ) is the maximum (resp. minimum) value of the objective of $\mathfrak{p}$.

We say that the problem $\mathfrak{p}$ has a polynomial time approximation scheme (PTAS) if for every $\epsilon>0$, there exists a $(1-\epsilon)$-approximation algorithm. 
For convenience of the notation, we call the feasible solution in Definition $4.1 \mathrm{a}$ $(1-\epsilon)$-approximation solution. Based on this definition, we discuss the conditions under which the problem (1.3) has PTAS.

First, we consider the following cubic polynomial optimization:

$$
\begin{aligned}
\min & \sum^{n, p, q} c_{i j k} x_{i} y_{j} z_{k} \\
\text { s.t. } & \|x\|=1,\|y\|=1,\|z\|=1, \\
& z \geq 0
\end{aligned}
$$

In this case, one can choose $z \in \Re_{+}^{q}$ from grid points $\left\{0, \sqrt{\frac{1}{d}}, \cdots, \sqrt{\frac{d-1}{d}}, 1\right\}$ such that $z_{1}^{2}+\cdots+z_{q}^{2}=1$, for some given integer $d$. They represent uniform grid points on the partial sphere $\left\{z \in \Re_{+}^{q}:\|z\|=1\right\}$. We call the chosen points feasible grid points. Then, the total number of such feasible grid points is $\left(\begin{array}{c}q+d-1 \\ d\end{array}\right)$ which is a polynomial in $q$ for any fixed integer $d \geq 1$.

For each feasible grid point $\bar{z}$, one can solve the maximum singular value of the matrix $C \bar{z}$ with its $(i, j)$ th entry being $(C \bar{z})_{i j}=\mathcal{C}_{i j k} \bar{z}_{k}$. It is well known that the singular value of matrix can be solved in polynomial time. Let $\bar{z}$ be the one among these grid points whose largest singular value is the greatest and let $\bar{x}, \bar{y}$ be the corresponding left-singular and right-singular vectors. Then, by Bomze and de Klerk [1, the feasible solution pair $(-\bar{x}, \bar{y}, \bar{z})$ returns a $\left(1-\frac{1}{d}\right)$-approximation solution to (4.1). Hence, the following result is established.

Theorem 4.1. There is a PTAS for solving problem (4.1).

Note that the variables $x, y$ and $z$ are equal "in rights", and so Theorem 4.1 is valid when we consider the case that $z \geq 0$ is replaced by $x \geq 0$ or $y \geq 0$. In fact, for the original optimization, if we know the sign of optimal vector $x^{*}$ or $y^{*}$ or $z^{*}$ in advance, then the PTAS can be modified slightly. For instance, for $A^{(i)}$ $(i=1, \cdots, n)$ as in (3.1), if all entries in matrix $A^{(i)}$ have the same sign pattern, then we have that

$$
|\mathcal{C} x y z|=\left|y^{\top}\left(\sum_{i=1}^{n} A^{(i)} x_{i}\right) z\right| \leq|y|^{\top}\left|\sum_{i=1}^{n} A^{(i)} x_{i}\right||z|,
$$

where $|\cdot|$ denotes the entries being the absolute value of corresponding entries. Hence the sign of the optimal solution $\left(x^{*}, y^{*}, z^{*}\right)$ can be known in advance, which leads to a PTAS. In a similar pattern, there exists a PTAS if each matrix $B^{(j)}$ or $C^{(k)}$ has the same sign.

Furthermore, note the fact that the number of sign patterns for $x, y$ and $z$ are at most $2^{n}, 2^{p}$ and $2^{q}$, respectively. Thus, if $\min \{n, p, q\}$ is fixed, then a PTAS for (1.3) can be obtained by solving subproblem (4.1) at most $2^{\min \{n, p, q\}}$ times, which leads to the following corollary.

Corollary 4.1. If $\min \{n, p, q\}$ is fixed, there exists a PTAS based on the grid sampling on simplex for solving (1.3).

On the other hand, combining with Corollary 3.7 and Theorem 4.1 in [8], we have the following result.

Corollary 4.2. If $\min \{n, p, q\}$ is fixed, then there exists a PTAS based on the sum of the square for solving (1.3). 


\section{Relative QuAlity Bound of (1.3)}

In this section, we present a quality bound of (1.3). To present our results, we begin with the classical definition of a relative quality bound:

Definition 5.1. Let $\mathfrak{A}$ be a polynomial time approximation algorithm for the minimization problem $\mathfrak{p}$. Let $0<E \leq 1$. For any instance of $\mathfrak{p}$, we say $\mathfrak{A}$ has a relative quality bound $0<E \leq 1$ for it, if the algorithm $\mathfrak{A}$ can find an upper bound $p$ such that

$$
\begin{cases}E \cdot p \leq p_{\min } \leq p, & \text { if } p_{\min } \geq 0 \\ p_{\min } \leq p \leq E \cdot p_{\min }, & \text { if } p_{\min }<0\end{cases}
$$

where $p_{\min }$ is the optimal value of $\mathfrak{p}$.

For simplicity, we call the bound $p$ in Definition $5.1 E$-bound.

Evidently, by this definition, the closer $E$ is to 1, the better the approximation algorithm will be. In general, Definition 5.1 is stronger than Definition 4.1 However, it is clear that both definitions are equivalent for odd order spherical optimization problems.

In the subsequent discussion, we will present a quality bound for (1.3) by finding the largest singular values of matrices.

Theorem 5.1. There exists a $\frac{1}{\sqrt{\min \{n, p, q\}}}$-bound for (1.3).

Proof. To get the relative quality bound, we rewrite (1.3) as

$$
\mathcal{C} x y z=\sum_{k=1}^{q}\left(x^{\top} C^{(k)} y\right) z_{k},
$$

for some suitable matrices $C^{(k)}$ as in (3.1).

For $k=1,2, \cdots, q$, the following problem can be solved in polynomial time by finding the largest singular value of matrix $C^{(k)}$ :

$$
\begin{array}{cl}
\max & x^{\top} C^{(k)} y \\
\text { s.t. } & \|x\|=1 \\
& \|y\|=1
\end{array}
$$

Let $k^{*}$ be the one whose objective value is the largest and let $x^{*}, y^{*}$ be the optimizer and let $z_{k^{*}}^{*}$ be -1 accordingly and the rest of entries 0 . Then for any feasible solution pair $(x, y, z)$, there holds

$$
\begin{aligned}
(\mathcal{C} x y z)^{2} & =\left(\sum_{k=1}^{q}\left(x^{\top} C^{(k)} y\right) z_{k}\right)^{2}=\sum_{i, j=1}^{q}\left(x^{\top} C^{(i)} y\right)\left(x^{\top} C^{(j)} y\right) z_{i} z_{j} \\
& \leq q \sum_{i=1}^{q}\left(x^{\top} C^{(i)} y\right)^{2} z_{i}^{2} \leq q \sum_{i=1}^{q}\left(x^{*} C^{\left(k^{*}\right)} y^{*}\right)^{2} z_{i}^{2} \leq q\left(x^{* \top} C^{\left(k^{*}\right)} y^{*}\right)^{2} .
\end{aligned}
$$

Furthermore, there holds

$$
\left(x^{* \top} C^{\left(k^{*}\right)} y^{*}\right)^{2}=\left(\mathcal{C} x^{*} y^{*} z^{*}\right)^{2} \leq \max _{\|x\|=\|y\|=\|z\|=1}(\mathcal{C} x y z)^{2} \leq q\left(x^{* \top} C^{\left(k^{*}\right)} y^{*}\right)^{2},
$$

which implies the result. 


\section{Practical method For solving (1.3)}

Since the approximation solution obtained in Theorem 5.1 may not be a KKT point of problem (1.3), we will propose a modified power method to get a 'good' solution of problem (1.3). Without loss of generality, we assume that $n \leq p \leq q$.

Algorithm 6.1.

- Initial step: Input tensor $\mathcal{C}$ and matrices $A^{(i)}$ as in 3.1).

- Substep 1: For each matrix $A^{(i)}$, compute the largest singular value $\lambda^{(i)}$ and its corresponding left-singular and right-singular vectors $y^{(i)}, z^{(i)}$.

- Substep 2: Choose the index $i_{0}$ with the singular value $\lambda^{\left(i_{0}\right)}$ being the largest among $\left\{\lambda^{(1)}, \lambda^{(2)}, \cdots, \lambda^{(n)}\right\}$.

- Substep 3: Let $y^{(0)}=y^{\left(i_{0}\right)}, z^{(0)}=z^{i_{0}}, x^{(0)}$ be the vector with $x_{i_{0}}^{(0)}=$ -1 and the rest of the entries being 0 . Let $l=0$.

- Iterative step: Compute matrix $\mathcal{C} x^{(l)} \in \Re^{p \times q}$ with its entries

$$
\left(\mathcal{C} x^{(l)}\right)_{j k}=\sum_{i=1}^{n} \mathcal{C}_{i j k} x_{i}^{(l)} .
$$

For given matrix $-\mathcal{C} x^{(l)}$, find the largest singular value $\lambda^{(l)}$ and its corresponding left-singular and right-singular vectors $y^{(l+1)}, z^{(l+1)}$. Let $x^{(\ln )}$ be the vector with entries

$$
\left(x^{(l n)}\right)_{i}=-\sum_{j=1}^{p} \sum_{k=1}^{q} \mathcal{C}_{i j k} y_{j}^{(l+1)} z_{k}^{(l+1)} .
$$

Take $x^{(l+1)}=\frac{x^{(l n)}}{\left\|x^{(l n)}\right\|}$ and $l=l+1$.

Obviously, Algorithm 6.1 includes two parts: the initial step and the iterative step. By means of Theorem 5.1 the initial step provides a lower bound satisfying

$$
\mathcal{C} x y z \geq-\sqrt{n} \mathcal{C} x^{(0)} y^{(0)} z^{(0)}, \forall\|x\|=\|y\|=\|z\|=1 .
$$

Furthermore,

$$
\mathcal{C} x^{(l+1)} y^{(l+1)} z^{(l+1)}=-\left\|\mathcal{C} y^{(l+1)} z^{(l+1)}\right\| \leq \mathcal{C} x^{(l)} y^{(l+1)} z^{(l+1)} \leq \mathcal{C} x^{(l)} y^{(l)} z^{(l)},
$$

which indicates that the sequence $\left\{\mathcal{C} x^{(l)} y^{(l)} z^{(l)}\right\}$ generated by Algorithm6.1 is nonincreasing. Based upon this observation, we establish the following convergence result.

Theorem 6.1. Suppose that the sequence $\left\{\left(x^{(l)}, y^{(l)}, z^{(l)}\right)\right\}$ generated by Algorithm 6.1 is infinite. Then each accumulation point of the sequence $\left\{\left(x^{(l)}, y^{(l)}, z^{(l)}\right)\right\}$ is a KKT point of the problem (1.3).

Proof. Since $\left\{\mathcal{C} x^{(l+1)} y^{(l+1)} z^{(l+1)}\right\}$ is non-increasing, together with the fact that $\mathcal{C} x y z$ is bounded over unit spheres, we know that $\left\{\mathcal{C} x^{(l+1)} y^{(l+1)} z^{(l+1)}\right\}$ converges and

$$
\lim _{l \rightarrow \infty}\left(\mathcal{C} x^{(l+1)} y^{(l+1)} z^{(l+1)}-\mathcal{C} x^{(l)} y^{(l)} z^{(l)}\right)=0
$$

To establish the result, we need the convergence of $\left\{\lambda^{(l)}\right\}$. 
From the obtained $\lambda^{(l)}$, it suffices to show the convergence of $\left\{x^{(l)}\right\}$. From (6.1), we have

$$
\mathcal{C} x^{(l+1)} y^{(l+1)} z^{(l+1)}-\mathcal{C} x^{(l)} y^{(l)} z^{(l)} \leq \mathcal{C}\left(x^{(l+1)}-x^{(l)}\right) y^{(l+1)} z^{(l+1)} \leq 0
$$

and

$$
\left\|\mathcal{C} y^{(l+1)} z^{(l+1)}\right\| \geq\left|\mathcal{C} x^{(0)} y^{0} z^{(0)}\right|>0 .
$$

So we can assert that $\left\|x^{(l+1)}-x^{(l)}\right\| \rightarrow 0$. Hence, we can assert that $\left\{x^{(l)}\right\}$ is convergent. As a result, $\left\{\lambda^{(l)}\right\}$ is also convergent.

Suppose that $\left(x^{*}, y^{*}, z^{*}\right)$ is an accumulation point of $\left(x^{(l)}, y^{(l)}, z^{(l)}\right)$. Without loss of generality, we assume that

$$
\lim _{l \rightarrow \infty} x^{(l)}=x^{*}, \quad \lim _{l_{t} \rightarrow \infty} y^{\left(l_{t}\right)}=y^{*}, \quad \lim _{l_{t} \rightarrow \infty} z^{\left(l_{t}\right)}=z^{*}, \quad \lim _{l \rightarrow \infty} \lambda^{(l)}=\lambda^{*} .
$$

From the Iterative step of Algorithm 6.1, we have

$$
\left\{\begin{aligned}
\mathcal{C} x^{(l)} y^{(l+1)} & =-\lambda^{(l)} z^{(l+1)} \\
\mathcal{C} x^{(l)} z^{(l+1)} & =-\lambda^{(l)} y^{(l+1)} \\
\mathcal{C} y^{(l+1)} z^{(l+1)} & =-\left\|\mathcal{C} y^{(l+1)} z^{(l+1)}\right\| x^{(l+1)} \\
\left\|x^{(l+1)}\right\| & =\left\|y^{(l+1)}\right\|=\left\|z^{(l+1)}\right\|=1
\end{aligned}\right.
$$

Then, it holds that

$$
\left\{\begin{aligned}
\mathcal{C} x^{*} y^{*} & =-\lambda^{*} z^{*} \\
\mathcal{C} x^{*} z^{*} & =-\lambda^{*} y^{*} \\
\mathcal{C} y^{*} z^{*} & =-\left\|\mathcal{C} y^{*} z^{*}\right\| x^{*} \\
\left\|x^{*}\right\| & =\left\|y^{*}\right\|=\left\|z^{*}\right\|=1
\end{aligned}\right.
$$

As a consequence, we have

$$
\lambda^{*}=\left\|\mathcal{C} y^{*} z^{*}\right\|=-\mathcal{C} x^{*} y^{*} z^{*},
$$

which completes the proof.

To illustrate performance of the procedure, let us consider two examples.

Example 6.1. This example is taken from [14]. Tensor $\mathcal{C}$ is a random low-rank $40 \times 30 \times 40$ tensor generated as the sum of 20 rank-one tensors, each rank-one tensor $x \otimes y \otimes z$ is generated with components of $x, y$ and $z$ uniformly distributed in $(0,1)$. Figure 1 shows the numerical results, where "inval" and "lval" denote the initial value and corresponding lower bound obtained by the initial step of Algorithm6.1. respectively. "val" denotes the value obtained by Algorithm 6.1. From Figure 1, we can claim that the algorithm performs well. 


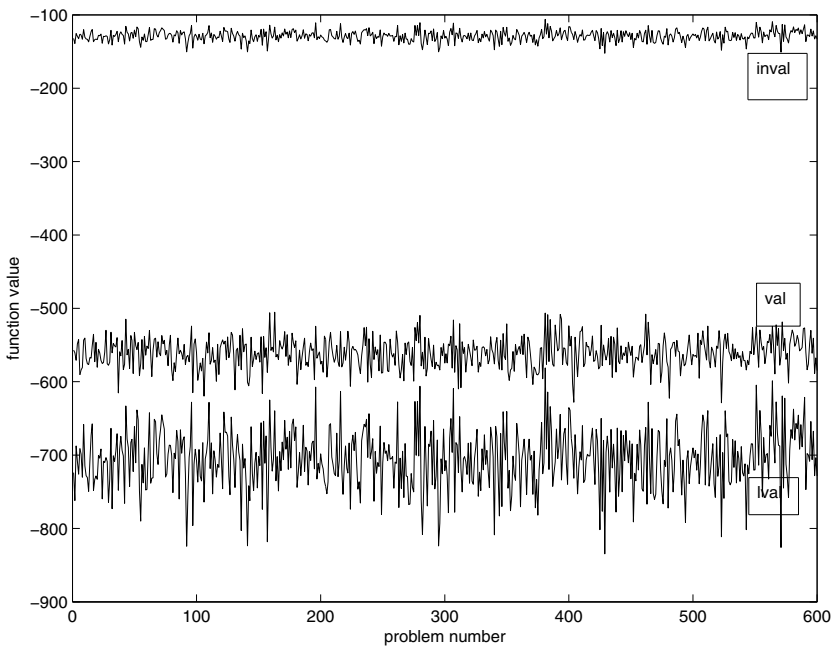

FiguRE 1. Numerical Results of Example 6.1

Example 6.2. Consider the problem (1.3) with $(n \times p \times q)$-dimensional tensor $\mathcal{C}$ whose entries are uniformly distributed in $(0,1)$.

The following table reports the average value of 100 problems, where "Dim" denotes the dimension of tensor $\mathcal{A C}$, "lval" denotes the average lower bound provided by the initial step and Theorem [5.1. "alval" denotes the average value obtained by Algorithm6.1. "CPU" denotes the average working time of the computer excluding the input/output time. "lval/alval" denotes the approximation ratio.

TABLE 1. Reports of Example 6.2.

\begin{tabular}{|c|l|l|l|l|}
\hline Dim & lval & alval & CPU & lval/alval \\
\hline$(3 \times 5 \times 10)$ & -6.7970 & -6.2303 & 0.0441 & 1.0910 \\
\hline$(5 \times 10 \times 30)$ & -20.5247 & -19.4263 & 0.0563 & 1.0565 \\
\hline$(10 \times 30 \times 50)$ & -63.2307 & -61.3158 & 0.0798 & 1.0312 \\
\hline$(30 \times 50 \times 100)$ & -197.9735 & -193.6653 & 0.4516 & 1.0222 \\
\hline$(50 \times 100 \times 300)$ & -618.4687 & -612.3631 & 3.8347 & 1.0100 \\
\hline$(100 \times 300 \times 500)$ & -1945.3 & -1936.4 & 22.5809 & 1.0046 \\
\hline
\end{tabular}

\section{REFERENCES}

1. I.M. Bomze and E. de Klerk, Solving standard quadratic optimization problems via linear, semidefinite and copositive programming. Journal of Global Optimization, 24 (2002), 163185. MR 1934026(2003i:90048)

2. J.F. Cardoso, High-order contrasts for independent component analysis. Neural Computation, 11 (1999), 157-192.

3. P. Comon, Independent component analysis, a new concept? Signal Processing, 36 (1994), 287-314.

4. S. He, Z. Li and S. Zhang, Approximation algorithms for homogeneous polynomial optimization with quadratic constraints. Mathematical Programming, 125 (2011), 353-383. MR.2733568

5. E. Kofidis and P.A. Regalia, On the best rank-1 approximation of higher-order supersymmetric tensors. SIAM Journal on Matrix Analysis and Applications, 23 (2002), 863-884. MR1896822 (2003a:15029) 
6. L. De Lathauwer, First-order perturbation analysis of the best rank- $\left(R_{1}, R_{2}, R_{3}\right)$ approximation in multilinear algebra. Journal of Chemometrics, 18 (2004), 2-11.

7. L. De Lathauwer, B. De Moor, J. Vandewalle, On the best rank-1 and rank- $\left(R_{1}, R_{2}, \ldots, R_{N}\right)$ approximation of higher-order tensor. SIAM Journal on Matrix Analysis and Applications, 21 (2000), 1324-1342. MR 1780276 (2001i:15034)

8. C. Ling, J. Nie, L. Qi and Y. Ye, Bi-quadratic optimization over unit spheres and semidefinite programming relaxations. SIAM Journal on Optimization, 20 (2009), 1286-1310. MR.2546345 (2010h:90087)

9. W.S. Lu and S.C. Pei, On optimal low-rank approximation of multidimensional discrete signals. IEEE Transacations on Circuits and Systems II, 45 (1998), 417-422.

10. Yu. Nesterov, Random walk in a simplex and quadratic optimization over convex polytopes. CORE Discussion Paper 2003/71, CORE, Catholic University of Louvain, Louvain-la-Neuve, Belgium, 2003.

11. J. Nie, Sum of squares methods for minimizing polynomial forms over spheres and hypersurfaces. preprint, 2009.

12. L. Qi, Eigenvalues of a real supersymmetric tensor. Journal of Symbolic Computation, 40 (2005), 1302-1324. MR.2178089 (2006j:15031)

13. L. Qi, F. Wang and Y. Wang, Z-Eigenvalue methods for a global optimization polynomial optimization problem. Mathematical Programming, 118 (2009), 301-316. MR2470793 (2009m:90111)

14. T. Zhang and G.H. Golub, Rank-1 approximation of higher-order tensors. SIAM Journal on Matrix Analysis and Applications, 23 (2001), 534-550. MR.1871328 (2002j:15039)

15. X. Zhang, C. Ling, L. Qi and E.X. Wu, The measure of diffusion skewness and kurtosis in magnetic resonance imaging. Pacific Journal of Optimization, 6 (2010), 391-404. MR 2668347

Department of Mathematics, School of Science, Tianjin University, Tianjin, 300072, CHINA.

E-mail address: xzzhang@tju.edu.cn

Department of Applied Mathematics, The Hong Kong Polytechnic University, Hung Hom, Kowloon, Hong Kong.

E-mail address: maqilq@polyu.edu.hk

Department of Management Science and Engineering, Stanford University, Stanford, CA94305 and The Hong Kong Polytechnic University, Hong Kong.

E-mail address: yinyu-ye@stanford.edu 